\title{
Effectiveness of Metoclopramide Combined with Dexamethasone and Granisetron alone in Preventing Post-operative Nausea and Vomiting in High Risk Patients Undergoing Laparoscopic Cholecystectomy
}

\section{Rahnuma Tasnim ${ }^{1 *}$, Debasish Banik ${ }^{2}$, Md. Abdur Rahim ${ }^{3}$, Shamima Akter ${ }^{4}$, Sahin Sultana Chowdhury ${ }^{5}$, Dilip Kumar} Bhowmik $^{6}$

\author{
${ }^{1}$ Medical Officer, Department of Anaesthesia, Analgesia and Intensive Care Medicine, Bangabandhu Sheikh Mujib Medical University, Dhaka, \\ Bangladesh \\ ${ }^{2}$ Professor, Department of anaesthesia, Analgesia and Intensive Care Medicine, Bangabandhu Sheikh Mujib Medical University, Dhaka, Bangladesh \\ ${ }^{3}$ Registrar, Department of Cardiac Anaesthesia, National Heart Foundation and Research Institute, Dhaka, Bangladesh \\ ${ }^{4}$ Classified Anaesthesiologist, Combined Military Hospital, Rongpur, Bangladesh \\ ${ }^{5}$ Medical Officer, Sheikh Hasina National Institute of Burn and Plastic surgery, Dhaka, Bangladesh \\ ${ }^{6}$ Associate Professor, Department of Anaesthesia, Analgesia and Intensive Care Medicine, Bangabandhu Sheikh Mujib Medical University, Dhaka, \\ Bangladesh
}

DOI: $10.36347 /$ sjams.2020.v08i09.019

| Received: 06.09.2020 | Accepted: 14.09.2020 | Published: 17.09.2020

*Corresponding author: Rahnuma Tasnim

Abstract

Original Research Article

Objective: In this study our main goal is to comparison of the effectiveness of metoclopramide combined with dexamethasone and granisetron alone in preventing post-operative nausea and vomiting in high risk patients undergoing laparoscopic cholecystectomy. Method: It is a cross sectional observational study on 120 patients who underwent laparoscopic cholecystectomy. The study period was July, 2017 to June, 2018. The study was conducted in the department of Anesthesia, Analgesia and Intensive care medicine of BSMMU. All the patients were chosen by purposive sampling. The study population was divided into two groups by simple lottery method. Group A patients got Granisetron (1mg iv single dose) and Group B patients got Metoclopramide and Dexamethason (Inj. Metoclopramide (10mg) and Inj. Dexamethasone (8mg)) just before induction. The observations were plotted into tabular and figure form. The categorical variable was analyzed by chi square test and the quantitative variables were analyzed by

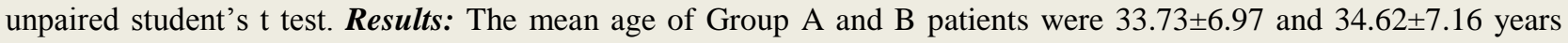
respectively. The sex ratio showed 21:39 and 15:45 in case of Male: Female respectively.out of 60 patients in each group PONV was reported in 20 (33.33\%) and 16(26.67\%) patients in Group A and Group B respectively. No PONV was reported in $40(66.67 \%)$ and $44(73.33 \%)$ patients in Group A and B respectively. 40(66.67\%) and 44(73.33\%) in Group A and B respectively showed no nausea and vomiting. 8(13.33\%) and 7(11.67\%) patients showed score 1 (nausea) in Group A and B respectively. On the contrary, 7(11.67\%) and 5(8.33\%) patients in Group-A and B respectively were categorized PONV score 2 (who experienced both nausea and vomiting. Only 5(8.33\%) and $4(6.67 \%)$ patients experienced repeated vomiting $\geq 2$ times who were categorized as PONV score 3. $(p=0.872)$. Among rescue antiemetic drug, analgesic drug use and postoperative hospital stay nothing but the hospital stay showed the statistically significant difference between the groups $(\mathrm{P}=0.000044)$. Conclusion: From our results we can conclude that, no statistically significant difference was observed between metoclopramide combined with dexamethasone group and granisetron alone except the granisetron from the point of view of short hospital stay.

Keywords: Metoclopramide, Dexamethasone, Granisetron, Cholecystectomy.

Copyright @ 2020: This is an open-access article distributed under the terms of the Creative Commons Attribution license which permits unrestricted use, distribution, and reproduction in any medium for non-commercial use (NonCommercial, or CC-BY-NC) provided the original author and source are credited.

\section{INTRODUCTION}

Post-operative nausea and vomiting (PONV) is one of the most unpleasant complications associated with anesthesia and surgery. It is the fifth commonest general complication of major abdominal surgery. It accounts for at least $25-30 \%$ of all cases [1]. The incidence of PONV is nearly $80 \%$ in high risk patients for PONV [2].

The aetiology of nausea and vomiting after surgery is multifactorial in origin. Age, menstrual cycle, type of surgery and anaesthetic procedure may influence PONV [3]. Many drugs have been tried to prevent or alleviate this problem. But most of the time it 
is difficult to predict, prevent or to treat. Plenty of antiemetic drugs are available which include anticholinergic drugs (Scopolamine, Atropine), Dopamine antagonist drugs (Promethazine, Prochlorperazine and Metoclopramide), antihistamine drugs (Diphenhydramine Hydroxine), 5HT3-receptor antagonists (Ondansetron, Granisetron, Dolasetron) and Steroids (Dexamethasone).

In spite of plenty of antiemetic drugs available no single drug is $100 \%$ effective against PONV. Because it is multifactorial in origin and there is no single stimulus for PONV. So in recent years interest has been focused on combination therapy.

Using Apfel's risk assessment tool, the level of risk can be quantified. Low risk patient score $0-1$, where there is a $10 \%$ to $20 \%$ possibility of them experiencing PONV. Moderate risk patient score 2, where there is a $40 \%$ possibility of this group experiencing PONV, and high risk patient score 3-4, their potential to experience PONV lies between $60 \%$ to $80 \%$. We include this high risk patient in our study whose Apfel's score 3-4. Estimating the individual's baseline risk for PONV can indicate who will most likely benefit from prophylactic antiemetic therapy. In general prophyxis in a patient already at low risk for PONV will not produce a clinically meaningful decrease in PONV incidence. On the other hand even a small decrease in PONV risk might be clinically desirable for patient where vomiting can cause significant medical harm [4]. PONV are commonly observed undesirable consequences of laparoscopic cholecystectomy where most of the patient is female who are prone to develop PONV.

The introduction of 5HT3 receptor antagonist in 1990s was heralded as a major advance in the treatment of PONV because of the absence of adverse effect that were observed with commonly used traditional antiemetics like delayed recovery, sedation or extrapyramidal symptoms [5]. Serotonin receptors of $5-\mathrm{HT}_{3}$ type are located peripharally on vegal nerve terminals and centrally in the chemoreceptor trigger zone of the area postrema.

Recently the antiemetic effect of Dexamethasone alone was demonstrated successfully in the patient of paediatric tonsillectomy and ambulatory Gynecological procedure [6]. Dexamethasone is also found to reduce pain and swelling following extraction of third molar tooth.

Pneumoperitonium needed for Laparoscopy has got direct effect on postoperative nausea and vomiting due to retention of $\mathrm{CO} 2$, which acts both centrally and peripherally (Strunin L- $6^{\text {th }}$ edition). After laparoscopic surgery discharge from hospital is sometimes delayed due to postoperative nausea and vomiting [7].
Dexamethasone is considered as one of the important anti-emetic. Adverse effects with a single dose of dexamethasone are extremely rare and generally minor. It is relatively inexpensive and easily available. The mechanism of dexamethasone-induced antiemesis is not fully understood, but central inhibition of prostaglandin synthesis and decrease in 5HT turnover in the central nervous system or changes in the permeability of the blood CSF barrier to serum proteins may be involved [8]. Surgery causes injury to the nerve endings. Pain sensitivity of the nociceptive neuron is further activated by certain peripheral chemical mediators. Among the mediators, the sensory nociceptors are mainly sensitized by prostaglandin, so drugs such as dexamethasone, which inhibit prostaglandin synthesis, may reduce inflammatory and sensory responses [9].

Metoclopramide acts at dopamine receptors in the stomach, upper intestine and CTZ. It enhances gastric emptying and lower esophageal sphinter pressure. Metoclopramide is still prescribed frequently as an antiemetic because of its easy availability and cost effectiveness.

With effective reduction of frequency of PONV early shifting of patients from recovery room to ward is possible. That can also reduce medical cost significantly. In this regard, granisetron may be an effective choice. So, to test its action in comparison to metoclopramide plus dexamethasone in preventing PONV; this research has been initiated.

In laparoscopic surgery carbon dioxide gas is introduced into abdominal cavity that creates a pressure locally in stomach. Besides for neurological stimulation of some anesthetic and analgesic drugs in chemoreceptor trigger zone postoperative nausea and vomiting are very much evident. Many drugs include 5HT3, dopaminergic, histaminic and NKI antagonists are being used now-a-days in this regard.

Laparoscopic surgery is a common operation usually performed as many as $42-72 \%$ patients [10]. This study will try to compare the anti-emetic effect of prophylactic granisetron versus metoclopramide combined with dexamethasone in high risk patients who will undergo laparoscopic cholecystectomy.

\section{OBJECTIVE \\ General objective}

Comparison of the effectiveness of metoclopramide combined with dexamethasone and granisetron alone in preventing post-operative nausea and vomiting in high risk patients undergoing laparoscopic cholecystectomy. 


\section{Specific Objective}

- To observe the need of rescue antiemetic and duration of post-operative hospital stay in metoclopramide with dexamethasone group.

- To observe the need of rescue antiemetic and duration of post-operative hospital stay in granisetron group.

- To compare the need of rescue antiemetic and duration of post-operative hospital stay between two groups.

\section{METHODOLOGY}

Study design: Cross sectional study.

Place of study: Department of Anaesthesia, Analgesia and Intensive Care Medicine, BSMMU, Dhaka.

Study Period: One year (July 2017-June 2018)

Study Population: Patient elected for laparoscopic cholecystectomy in the Department of Surgery, BSMMU.

Sampling method: Purposive sampling

Sample size: 120 patients were enrolled for the study, 60 in each group. Each 60 cases were enrolled in Group A and Group B by simple lottery method.

\section{Eligibility CRiteria Inclusion Criteria}

- Patients who underwent laparoscopic cholecystectomy under general anesthesia as a high risk patient for PONV (Apfel's score 34).

- Patients with American Society of Anesthesiologists (ASA) physical status I \& II.

\section{Exclusion Criteria}

- Patients with American Society of Anesthesiologists (ASA) physical status $>$ II.

- $\quad$ BMI $>30$

- $\quad$ Age $<17$ or $>70$ years.

- Renal or liver failure

- Concomitant disease with nausea and vomiting.

- Use of anti-emetic in 24 hours before surgery

- Antidepressant use

- Pregnancy and breastfeeding

- Conversion from laparoscopic cholecystectomy to open cholecystectomy.

- $\quad$ Patients with diabetes mallitus

\section{Procedure of data collection}

A data sheet and a consent form was prepared, sample was selected on the basis of inclusion and exclusion criteria, questionnaire was filled with informed written consent, interview was taken. Apfel's risk assessment score includes 4 points. History of
PONV or motion sickness, nonsmoker, female and postoperative opioid treatment is planned. Each point includes 1 point. We took Apfel's score 3-4 in this study. The whole sample size was divided into 2 groups-Groups A and Group B. Group A patients got Granisetron and Group B patients got Metoclopramide and Dexamethason just before induction. Just before induction Granisetron 1mg iv single dose in Group A patients and Metoclopramide 10mg plus Dexamethasone $8 \mathrm{mg}$ in Group B patients was given. At end of operation patient was reversed accordingly with neostigmine $0.05 \mathrm{mg} / \mathrm{kg}$ plus atropine $0.02 \mathrm{mg} / \mathrm{kg}$ and recovery. Intraoperative and postoperative monitoring was by NIBP, ECG, SpO2.In the recovery room postoperative analgesia was provided with pathedine (1-1.5 $\mathrm{mg} / \mathrm{kg}$ ) stat and Ketorolac Tromethamine 30mg intramuscularly 8 hourly on complaining pain and repeated in all patients when necessary. Patients were evaluated for nausea and vomiting in the first 24 hours, nausea was rated on the VRS (visual rating score) grade $\left(\mathrm{G}_{0}\right.$-no nausea, $\mathrm{G}_{1}$-nausea, $\mathrm{G}_{2}$-nausea with vomiting, $\mathrm{G}_{3}$-repeated vomiting) and number of vomiting episodes was recorded. Patients were carefully observed for any adverse effect like, drowsiness, flushing or any extrapyramidal symptoms. Data was gathered, edited, decorated in SPSS version 23. Data were analyzed. The observations were plotted into tabular and figure form.

\section{Statistical Analysis}

All data were checked and edited after collection and presented in graph, chart and tabulated form where applicable. Frequency of distribution and normal distribution of all continuous variables were calculated. Cross tabulation was prepared and a comparison was made between the respondents from different age, sex, clinical features. Chi square test was used to analyze the qualitative variables like frequency of PONV, frequency of rescue antiemetics and drug related adverse effects. Finally statistical analysis was carried out by using the statistical package for social sciences version 23 for windows. $\mathrm{P}$ values $<0.05$ was considered as statistically significant.

\section{RESULTS}

Table-1 shows that the mean age of Group A and B patients were 33.75 6.97 (age range: 28-69) years and 34.66 \pm 7.16 (age range: 35-70) years respectively. The male to female ratio revealed that 21:39 and 15:45 in the both groups respectively. The mean weight of the patients are a little higher of Group A patients than Group B patients $(58.73 \pm 12.73 \mathrm{~kg}$ vs $56.77 \pm 10.39 \mathrm{~kg}$ ) respectively. The similar scenario was observed in case of BMI $(23.16 \pm 1.79$ vs $22.35 \pm 1.31)$. The mean duration of anesthesia in Group A was $55.16 \pm 18.77$ minutes whereas the same in Group B was $53.76 \pm 18.39$ minutes. The mean duration of surgery in Group A was $52.65 \pm 16.54$ minutes and in group B $51.31 \pm 17.5$ minutes 
Table-1: Baseline characteristics of the study patients ( $N=120 ; 60$ in each group)

\begin{tabular}{|l|c|c|}
\hline \multicolumn{1}{|c|}{ Characteristics } & $\begin{array}{c}\text { Group-A } \\
(\mathbf{n = 6 0})\end{array}$ & $\begin{array}{c}\text { Group-B } \\
(\mathbf{n = 6 0})\end{array}$ \\
\hline Mean age \pm SD (in years) & $33.73 \pm 6.97$ & $34.62 \pm 7.16$ \\
\hline Age range (in years) & $28-69$ & $35-70$ \\
\hline Sex ratio (M:F) & $21: 39$ & $15: 45$ \\
\hline Apfel's score (n, \%) & & \\
\hline 3 & $33(55 \%)$ & $29(48.33 \%)$ \\
\hline 4 & $17(28.33 \%)$ & $31(51.67 \%)$ \\
\hline Mean weight \pm SD (in kg) & $58.73 \pm 12.73$ & $56.77 \pm 10.39$ \\
\hline Mean BMI \pm SD (in kg/m $\left.{ }^{2}\right)$ & $23.16 \pm 1.79$ & $22.35 \pm 1.31$ \\
\hline Duration of anesthesia Mean \pm SD (in minutes) & $55.16 \pm 18.77$ & $53.76 \pm 18.39$ \\
\hline Duration of surgery Mean \pm SD (in minutes) & $52.65 \pm 16.54$ & $51.31 \pm 17.5$ \\
\hline
\end{tabular}

Figure-1 shows that out of 60 patients in each group PONV was reported in $20(33.33 \%)$ and 16(26.67\%) patients in Group A and Group B respectively. No PONV was reported in 40
$(66.67 \%)$ and $44(73.33 \%)$ patients in Group A and B respectively.

Incidence of PONV ( $=120 ; 60$ in each group)

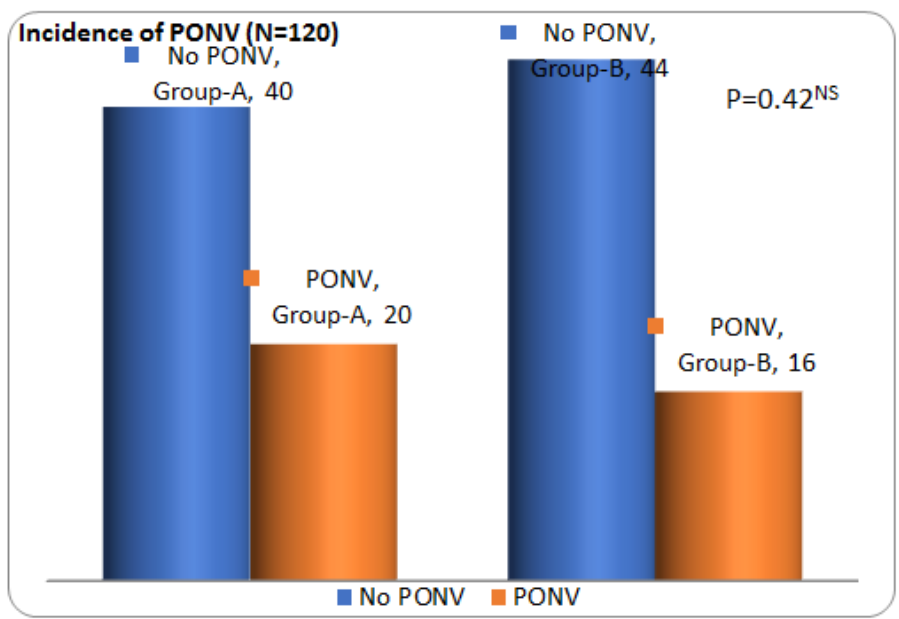

Fig-1: Distribution of patients according to incidence of $P O N V(N=120 ; 60$ in each group)

NS: Not significant; P-value was calculated by chi square test; $P$ was significant at $<0.05$.

Table-2 shows that out of 60 patients of each group, 40(66.67\%) and 44(73.33\%) in Group A and B respectively showed no nausea and vomiting. $8(13.33 \%)$ and $7(11.67 \%)$ patients showed score 1 (nausea) in Group $\mathrm{A}$ and $\mathrm{B}$ respectively. On the contrary, $7(11.67 \%)$ and $5(8.33 \%)$ patients in Group-A and $\mathrm{B}$ respectively were categorized a PONV score 2 (who experienced both nausea and vomiting. Only $5(8.33 \%)$ and $4(6.67 \%)$ patients experienced repeated vomiting $\geq 2$ times who were categorized as PONV score 3 . None of the scoring difference between the groups showed statistically significant $(\mathrm{P}=0.872)$.

Table-2: PONV score assessment ( $\mathrm{N}=120 ; 60$ in each group)

\begin{tabular}{|c|c|c|c|}
\hline PONV score & $\begin{array}{c}\text { Group-A } \\
(n=60)\end{array}$ & $\begin{array}{c}\text { Group-B } \\
(n=60)\end{array}$ & P-value \\
\hline G0: No nausea/vomiting & $40(66.67 \%)$ & $44(73.33 \%)$ & \multirow[t]{4}{*}{$0.872^{\mathrm{NS}}$} \\
\hline GI: Nausea & $8(13.33 \%)$ & $7(11.67 \%)$ & \\
\hline G2: Nausea with vomiting & $7(11.67 \%)$ & $5(8.33 \%)$ & \\
\hline G3: Repeated vomiting $\geq 2$ times & $5(8.33 \%)$ & $4(6.67 \%)$ & \\
\hline
\end{tabular}

P-value was calculated by chi square test NS: Not significant

P-value was significant at $<0.05$

PONV: Post-operative nausea and vomiting

Figure-II shows that Group a patients showed mostly the PONV in later period whereas the opposite was seen for Group B. 


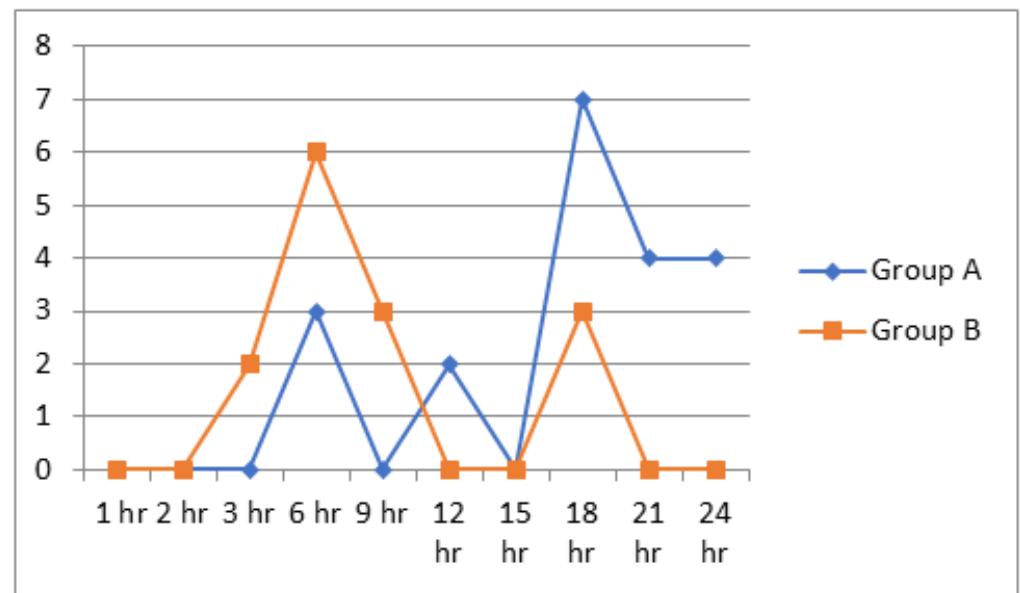

Fig-II: Distribution of PONV in relation to different time period of postoperative follow up (N=120; 60 in each group)

\section{Incidence of side effects $(\mathrm{N}=120 ; 60$ in each group)}

Table-3 shows that out of 60 patients in each group only 2 patients in Group A experienced complications. Between these 2(3.33\%) patients, $1(1.67 \%)$ had only dizziness and another $1(1.67 \%)$ had both headache and dizziness. On the contrary, out of 60 patients in Group B, 4(6.67\%) patients experienced complications. Among these 4 patients $2(3.33 \%)$ had only headache and rest were suffering from both headache and dizziness? No side effect showed statistically significant difference between the groups $(\mathrm{P}=0.731)$.

Table-3: Distribution of patients according to incidence of side effects ( $N=120 ; 60$ in each group)

\begin{tabular}{|l|c|c|c|}
\hline Side effects & $\begin{array}{c}\text { Group-A } \\
(\mathbf{n = 6 0})\end{array}$ & $\begin{array}{c}\text { Group-B } \\
(\mathbf{n}=\mathbf{6 0})\end{array}$ & P-value \\
\hline Headache & $1(1.67 \%)$ & $4(6.67 \%)$ & $0.731^{\mathrm{NS}}$ \\
\hline Dizziness & $2(3.33 \%)$ & $2(3.33 \%)$ & \\
\hline
\end{tabular}

P-value was calculated by chi square test NS: Not significant

P-value was significant at $<0.05$

Table-4 shows that among rescue antiemetic drug, analgesic drug use and postoperative hospital stay nothing but the hospital stay showed the statistically significant difference between the groups $(\mathrm{P}=0.000044)$.

Table-4: Postoperative outcome ( $N=120 ; 60$ in each group)

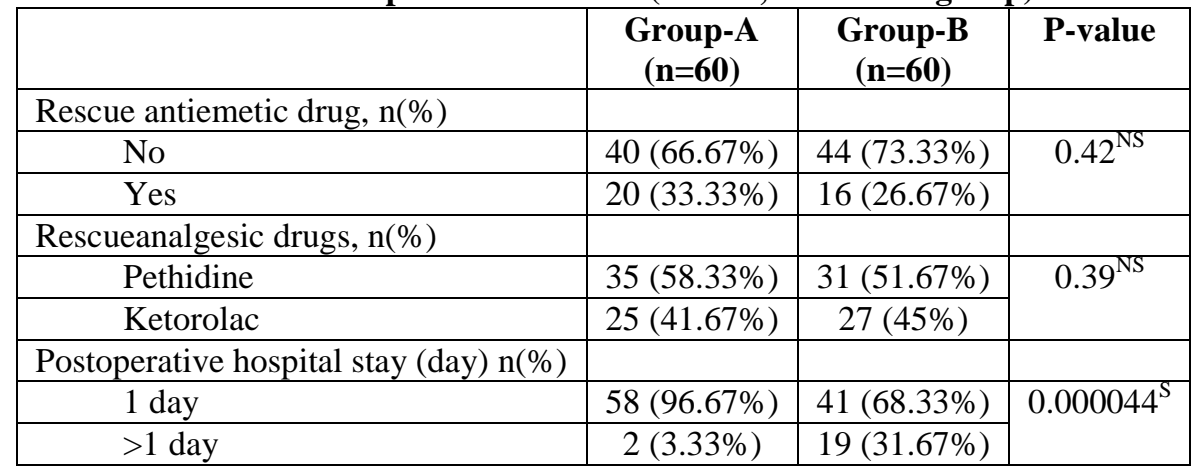

P-value was calculated by chi square test (categorical variable) and student's t test (quantitative variable)

NS: Not significant

S: Significant

P-value was significant at $<0.05$

\section{DisCUSSION}

This study we compared the effectiveness of metoclopramide combined with dexamethasone and granisetron alone in patients undergoing laparoscopic cholecystectomy.
In this study, the sample population was divided into 2 groups where 60 in each. They were namely Group A and B. Group A patients were treated by Inj. Granisetronei/v (1 mg) whereas Group B patients were treated by combination of Inj. Metoclopromide $(10 \mathrm{mg})$ plus Inj. Dexamethasone $(8$ $\mathrm{mg})$. Metoclopramide and Dexamethasone combination 
could not reveal the statistically significant superiority over Granisetrone. Because PONV was reported in 20 $(33.33 \%)$ and $16(26.67 \%)$ patients in Group A and Group B respectively $(\mathrm{p}=0.42)$. Our findings revealed that our PONV cases in Group B was almost double than those [11].

Almost similar numbers of patients were categorized as G1 (13.33\% vs $11.67 \%)$, G2 (11.67\% vs $8.33 \%)$ and $\mathrm{G} 3(8.33 \%$ vs $6.67 \%)$ in Group A and B respectively. So, there was no statistically significant difference found in these two categories $(\mathrm{p}=0.872)$. But the reverse was observed in a previous Bangladeshi study [12]. In this study, they compared Ondensetrone and Ondensetrone plus Dexamethasone. On the contrary, the reverse scenario was also observed in a study done by Rudra A and his colleagues where they compared Granisetrone plus Dexamethasone with Granisetrone alone. But the comparative study like ours is done for the first time in Bangladesh. So, we can conclude that Granisetrone versus Metoclopramide plus Dexamethasone is still to be judged in a large cohort study to determine the superiority in preventing PONV after laparoscopic cholecystectomy.

In this study we found 20 patients $(33.33 \%)$ got rescue antiemetics in group A and 16 (26.67\%) patients got rescue antiemetics in Group B. In another study found that rescue antiemetics required in 5 patients $(7.7 \%)$ who got prochlorperazine and 4 patients (6.2\%) who got cyclizine and 7 patients $(10.8 \%)$ who got ondansetron [13]. In our study the high incidence of PONV is due to high risks patients were included who were prone to develop PONV. In another study showed that patients treated with levosulpiride need less rescue antiemetics ( 7 patients) than control group (16 patients) following laparoscopic cholecystectomy. This may be due to that control group who did not receive any medication.

The mean age of the patients in Group A and B were $33.75 \pm 6.97$ years and $34.66 \pm 7.16$ years respectively. These were in line with the findings of $\mathrm{L}$. Sanjowal and his colleagues' reports Sanjowal [12].

The adverse effects of drug showed that headache, dizziness were common in both groups though they are very few in numbers. Here we did not find any statistically significant difference between the groups $(\mathrm{p}=0.731)$. The similar findings were also observed between the same drugs regimen in the study done by Daria U and Kumar [14].

Postoperative outcome of was compared between the groups regarding requirements of rescue antiemetic drugs, analgesic drug use, postoperative hospital stay. Among them only post-operative hospital stay showed the better results in case of Granisetrone received patients in comparison to Metoclopramide plus Dexamethasone received patients. $(p=0.000044)$ In our study we have found in Group A duration of hospital stay is 1 day in 58 patients $(96.67 \%)$ and more than 1 day in 2 patients $(3.33 \%)$ which is significant. It may be due to longer duration of granisetron.

\section{CONCLUSiON}

No statistically significant difference was observed between metoclopramide combined with dexamethasone group and granisetron alone except the granisetron from the point of view of short hospital stay.

\section{REFERENCES}

1. Cohen MM, Duncan PG, DeBoer DP, Tweed WA. The postoperative interview: assessing risk factors for nausea and vomiting. Anesthesia \& Analgesia. 1994 Jan 1;78(1):7-16.

2. Apfel CC, Läärä E, Koivuranta M, Greim CA, Roewer N. A simplified risk score for predicting postoperative nausea and vomiting conclusions from cross-validations between two centers. Anesthesiology: The Journal of the American Society of Anesthesiologists. 1999 Sep 1;91(3):693-

3. Lerman J. Surgical and patient factors involved in postoperative nausea and vomiting. British Journal of Anaesthesia. 1992 Jan 1;69:24S-.

4. Apfel CC, Kranke P, Katz MH, Goepfert C, Papenfuss T, Rauch S, Heineck R, Greim CA, Roewer N. Volatile anaesthetics may be the main cause of early but not delayed postoperative vomiting: a randomized controlled trial of factorial design. British journal of anaesthesia. 2002 May 1;88(5):659-68.

5. Paxton LD, McKay AC, Mirakhur RK. Prevention of nausea and vomiting after day case gynaecological laparoscopy: a comparison of ondansetron, droperidol, metoclopramide and placebo. Anaesthesia. 1995 May;50(5):403-6.

6. Splinter WM, Roberts DJ. Dexamethasone decreases vomiting by children after tonsillectomy. Anesthesia \& Analgesia. 1996 Nov 1;83(5):913-6.

7. Bisgaard T, Klarskov B, Kehlet H, Rosenberg J. Preoperative dexamethasone improves surgical outcome after laparoscopic cholecystectomy: a randomized double-blind placebo-controlled trial. Annals of surgery. 2003 Nov;238(5):651.

8. Altman DF. Drugs used in gastrointestinal diseases. Basic and clinical pharmacology, 8th edn. McGraw Hill, San Francisco. 2001:1070-1.

9. Ferreira SH, Vane JR. Mode of action of antiinflammatory agents which are prostaglandin synthetase inhibitors. InAnti-inflammatory drugs 1979 (pp. 348-398). Springer, Berlin, Heidelberg.

10. Fujii Y, Tanaka H, Toyooka H. RETRACTED ARTICLE: Granisetron reduces the incidence and severity of nausea and vomiting after laparoscopic cholecystectomy. Canadian journal of anaesthesia. 1997 Apr 1;44(4):396. 
11. Nesek-Adam V, Grizelj-Stojčić E, Rašić Ž, Čala Z, Mršić V, Smiljanić A. Comparison of dexamethasone, metoclopramide, and their combination in the prevention of postoperative nausea and vomiting after laparoscopic cholecystectomy. Surgical endoscopy. 2007 Apr 1;21(4):607-12.

12. Sanjowal L, Biswas SK, Saha JC. Comparison of ondansetron used alone and in combination with dexamethasone as a prophylaxis for postoperative nausea and vomiting following laparoscopic cholecystectomy. Faridpur Medical College Journal. 2014;9(1):3-6.
13. Malak AM, Shameem A, Ali CA, Qureshi MA. Comparison of ondansetron, cyclizine and prochlorperazine for PONV prophylaxis in laparoscopic cholecystectomy. Anaesthesia, Pain \& Intensive Care. 2017:453-7.

14. Usha D, Vinod K. Qualitative comparison of metoclopramide, ondansetron and granisetron alone and in combination with dexamethasone in the prevention of postoperative nausea and vomiting in day care laparoscopic gynaecological surgery under general anaesthesia. Asian J Pharm Clin Res. 2012;5:165-7. 